Journal of the Mathematical Society of Japan Vol. 1, No. 1, Sept., 1948.

\title{
On algebraic Lie groups and algebras.
}

\author{
Yozô Matsúshima.
}

(Received Oct. 25, 1947.)

\section{Introduction.}

Recently C. Chevalley and H. F. Tuan obtained an interesting characterization of the Lie algebras of algebraic Lie groups of matrices ${ }^{1)}$. Using the notion of replicas ${ }^{2)}$ of matrices, they introduced namely the concept of algebraic Lie algebras of matrices; A Lie algebra $\mathbb{L}$ of matrices over a field $P$ is called linear algebraic ( $l$-algebraic $)^{3)}$ if every replica of each $A \in \mathbb{Z}$ belongs also to $\mathfrak{R}$. It was shown by them that, if $P$ is the field of complex numbers, the lialgebraicity is the characteristic property of the Lie algebras of algebraic Lie groups of matrices. The notion has been extended recently by M. Gotô to general, not necessarily matric, Lie algebras ${ }^{4}$. Namely a Lie algcbra is called algebraic if its adjoint representation is l-algebraic. Then he proved that any algebraic Lie algebra over a fiele of characterirtic zero is isomorphic with an $l$-algebraic Lie algebra of matrices. In this note we shall first prove some results on $l$-algebraic Lie algebras. Most of these results have, been obtained by $C$. Chevalley and $\mathrm{H}$. F. Tuan, but our methods will be somewhat different from theirs. Then we shall characterize the Lie groups of algebraic Lie algebras. over the field of complex numbers. We show that the integrated groups of such Lie algebras are algebraic groups in the sense that the functions which define the multiplication of group elements are algebraic functions of suitably chosen parameters of the group. This result follows also from the above mentioned result of. M. Gotô, but our proof is a more direct one. The converse of this proposition has been already proved by L. Maurer ${ }^{\prime 5)}$ and thus we obtain a characterization of the Lie groups of algebraic Lie algebras. The writer is grateful to Mr. M. Gotô for his friendly cooperation.

1. Let $P$ be a field of characteristic zero. For simplicity we call a. 'nilpotent matrix an $n$-matrix, a matrix with simple elementary divisors an $s$-matrix, and an $s$-matrix whose characteristic roots are all rational numbers an $r$-matrix. Let $A$ be a matrix with coefficients in $P$ and $P$ be algebraically closed. In a previous note ${ }^{6)}$, we showed that we may represent $A$ in the form 


$$
A=A^{0}+\lambda_{1} A^{1}+\ldots \ldots+\lambda_{k} A^{k},
$$

where $\lambda_{1}, \ldots \ldots, \lambda_{k}$ are the characteristic roots of $A$ which are linearly independent with respect to the field $R$ of rational numbers, $A^{0}$ is an $n$-matrix and $A^{1}, \ldots \ldots, A^{k}$ are $r$-matrices such that $A^{i}(i=0,1, \ldots \ldots, k)$ are commutative with each other and form a linear basis for replicas of $A$. Now let

$$
A=B^{0}+\xi_{1} B^{1}+\ldots \ldots+\xi_{j} B^{3}
$$

be any decomposition of $A$ into an $n$-matrix $B^{0}$ and $r$-matrices $B^{j}$ such that $B^{l}(l=0,1, \ldots \ldots, j)$ are commutative with each other and $\xi_{i}(i=1, \ldots \ldots$, $j)$ are linearly independent over $R$. Then $B^{6}(i=0,1, \ldots \ldots, j)$ are replicas of $A$ and any replica of $A \cdot$ is a linear combination of $B^{i}{ }^{i)}$. For, we see first from (1) and (2) that

$$
A^{0}=B^{0}, \quad \lambda_{1} A^{1}+\ldots \ldots+\lambda_{k} A^{k}=\xi_{1} B^{\natural}+\ldots \ldots+\xi_{j} B^{j} .
$$

We may assume that $A^{i}$ and $B^{i} .(i \neq 0)$ are all diagonal matrices ${ }^{8)}$. Let

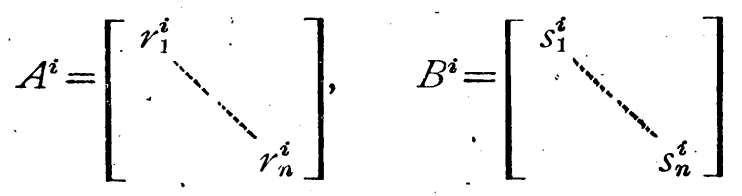

where $r_{j}^{i}$ and $s_{j}^{i}$ are rational numbers. Denote by $\mathfrak{M}$ a linear space spanned by $\lambda_{1}, \ldots \ldots, \lambda_{k}, \xi_{1}, \ldots \ldots, \xi_{j}$ over $R$, Let $\mu_{1}, \ldots \ldots, \mu_{m}$ be a basis of $\mathfrak{M}$ over $R$ such that $\mu_{i}=\lambda_{i}$ for $i=1, \ldots \ldots, k$. Let.

$$
\xi_{i}=\sum_{u=1}^{m} p_{i}^{n} \mu_{u}, \quad p_{i}^{n} \in R(i=1, \ldots \ldots, j)
$$

Then

$$
\sum_{i=0}^{j} \hat{\xi}_{i} s_{t}^{i}=\sum_{i=1}^{j} \sum_{u=1}^{m} p_{i}^{u} \mu_{u} s_{t}^{i}=\sum_{u=1}^{m} \mu_{u} \sum_{i=1}^{j} p_{i}^{u} s_{t}^{i}=\sum_{u=1}^{k} \mu_{u} r_{t}^{u}
$$

Hence

Therefore

$$
r_{t}^{u}=\sum_{i=1}^{j} p_{i}^{u} s_{t}^{t} \quad(u=1, \ldots \ldots, k ; t=1, \ldots \ldots, n)
$$

$$
A^{u}=\sum_{i=1}^{j} p_{i}^{u} B^{i} \quad(u=1, \ldots \ldots, k)
$$

We may prove by the same argument that $B^{i}$ are linear combinations of $A^{i}$ and this proves our assertion. We call in the following a decomposition (2) of a matrix $A$ a canonical decomposition. 
We denote by $g r(n, P)$, the Lie algebra of all matrices of degree $n$ with coefficients in the field $P$.

Lemma 1. Let $A, X \operatorname{egl}(\dot{n}, P)$. If $A X=X A$, then every replica of $A$ commutes with $X$.

Proof. We may assume that $P$ is algebraically closed. Let (1) be the caronical decomposition of $A$. Since $A$ commutes with $X$, every eigenspace of $A$ is invariant under $X$. As each $A_{i}(i=1, \ldots \ldots, k)$ has only one characteristic root and in fact represented by a scalar matrix in each of these eigen-spaces of $A^{9)}, A^{t}$ commutes with $X$. Hence $A^{0}$ commutes also with $X$, and so does every replica of $A$, since it is a linear combination of $A^{i}$.

Lemma 2. Let $\mathfrak{Q}$ be a Lie subalgebra of $\operatorname{gl}(n, P)$, where $P$ is of chara:teristic zero. Then the radical $\mathfrak{R}_{1}$ of the derived algebra $\mathfrak{Q}^{\prime}$ of $\mathfrak{Q}$ is composed only of nilpotent matrices.

Proof. Let $K$ be an algebraically closed field. containing $P$. We will show that every element of the radical $\mathfrak{R}_{1}$ of the derived algebra $\mathfrak{L}^{\prime}$ of any Lie algebra (not necessarily of matrices) $\mathfrak{L}$ over $P$ is represented by zero matrix in any (absolutely) irreducible representation of $\mathbb{R}$ in $K$. Any irreducible representation of $\mathfrak{L}$ in $K$ may be extended to the irreducible representation $\mathfrak{D}$ of $\mathfrak{R}_{K}{ }^{10)}$.

Let $\mathfrak{A}$ be the kernel of this representation $\mathfrak{D}$ of $\mathfrak{L}_{k}$. As $\mathfrak{D}$ is absolutely irreducible, $\left(\mathfrak{D}\right.$ is the direct sum of the semi-simple ideal $\left(\dot{D}^{\prime}\right)$ and the one-dimensional center ${ }^{11}$.

Since

$$
\mathfrak{R}_{K} / \mathfrak{A} \cong \mathfrak{D}
$$

$\mathfrak{l}_{K} / \mathfrak{U}$ must have the same structure and. we have

$$
\mathfrak{Q}_{\boldsymbol{K}} / \mathfrak{U}=\left(\mathfrak{Q}_{k} / \mathfrak{U}\right)^{\prime}+(\mathfrak{3} / \mathfrak{U}),
$$

where $\left(\mathfrak{L}_{K} / \mathfrak{U}\right)^{\prime}$ is the derived algebra of $\mathfrak{L}_{K} / A$ which is semi-simple and $3 / \mathfrak{A}$ is the one-dimensional center of $\mathfrak{Q}_{K^{\prime}} / \mathfrak{A}$. 'Since

$$
\left(\mathfrak{R}_{K} / \mathfrak{A}\right)^{\prime}=\left(\mathfrak{Q}_{K}^{\prime}, \mathfrak{U}\right) / \mathfrak{A} \cong \mathfrak{R}_{k}{ }^{\prime} / \mathfrak{R}_{K^{\prime}} \cap \mathfrak{U},
$$

$\mathfrak{Q}_{K}^{\prime} / \mathfrak{R}_{R^{\prime}} \cap \mathfrak{A}$ is semi-simple and $\mathfrak{Q}_{K^{\prime}} \cap \mathfrak{A}$ must contain the radical of $\mathfrak{Q}_{K^{\prime}}$. But since $\mathfrak{R}_{1 K}$ is contained in the radical of $\mathfrak{R}_{\mathbb{K}}{ }^{\prime} ; \mathfrak{R}_{1}$ is contained in $\mathfrak{A}$. Thus every element of $\mathfrak{R}_{1}$ is represented by zero matrix in any absolutely irreducible representation of $\mathfrak{L}$. Now, let $\mathfrak{Q}$ be $a^{\circ}$ Lie algebra of matrices. 
Then we may reduce $\mathbb{Q}$ in $K$ to the form

$$
\mathfrak{L}=\left[\begin{array}{lllll}
\mathfrak{L}_{1} & & & \\
& \mathfrak{R}_{2} & & \\
& & \ddots & \\
& * & & \mathfrak{Z}_{k}
\end{array}\right],
$$

where $\Omega_{i}(i=1,2, \ldots \ldots, k)$ are absolutely irreducible. Hence we see from the above consideration that every matrix of $\mathfrak{R}_{1}$ is nilpotent.

Remark. By-considering the adjoint representation, we may prove that the radical of the derived algebra of any Lie algebra (not necessarily of matrices) over $P$ is nilpotent ${ }^{12)}$.

Lemma 3. Let- $\mathfrak{Q}$ be a Lie algebra of matrices composed only of nilpotent matrices and $A$ be a matrix which admits as its invariants all the tensor invariants of $\mathfrak{L}$. Then $A$ belongs to $\mathfrak{L}$.

This lemma was obtained by I. Ado, and M. Gotô ${ }^{13)}$ gave an elementary proof using the notion of the replicas of matrices.

Lemma $4^{i 4)}$. Let $\mathfrak{M}$ be a vector space over an algebraically closed field $K$ and $A$ a linear transformation on $\mathfrak{M}$. Further let $\tilde{\mathfrak{M}}$ be the direct sum of some tensor spaces and $\widetilde{\mathfrak{N}}$ a subspace of $\tilde{\mathfrak{M}}$ which is invariant under $A$. We denote by $\tilde{A}$ the matrix of linear transformation which is induced by $A$ in $\tilde{\mathfrak{R}}$. Then $\widetilde{\mathfrak{N}}$ is invariant under all reprlicas of $A$ and the matrices of linear transformations which are induced by the replicas of $A$ in $\widetilde{\mathfrak{N}}$ are replicas of the matrix $\tilde{A}$. Further any replica of $\tilde{A}$ is induced conversely by a replica of $A$ in $\widetilde{\mathfrak{R}}$.

Lemma.5. Let $P$ be a field, $K$ an extension field of $P$ and $A$ a matrix in $\mathfrak{g l}(n, F)$. Then there exist replicas $A_{i}(i=1,2, \ldots \ldots, k)$ of $A$ in $\mathrm{gl}(n, P)$ such that every replica of $A$ in $\mathrm{gl}(n, K)$ is the form $\alpha_{1} A_{1}+\ldots \ldots$ $+\alpha_{k} A_{k}$ with $\alpha_{i} \in K$.

Proof. Let. $\mathfrak{I}_{r, s}$ be the space of $r$-times contravariant and $s$-times covariant tensors over $K$, and $\mathfrak{N}_{r, s}$ the subspace of all tensor invariants of $A$ in $\mathfrak{I}_{r, s}$. Let $\mathfrak{\Omega}_{p}$ be the set of all matrices in $\mathfrak{g l}(n, K)$ which admit as their invariants all tensors in $\mathfrak{N}_{r, s}$ with $r, s \leqq p$. Then $\mathfrak{\Omega}_{p}$ form a monotone decreasing series of linear spaces over $K$. whose intersection is the set $\mathcal{Q}$ of all replicas of $A$. Hence there exists an integer $m$ such that

$$
\mathfrak{R}_{m}=\mathfrak{R}_{m+1}=\ldots \ldots=\mathfrak{R} .
$$

On assuming $A \epsilon \mathfrak{g l}(n, P)$, we see from the theory of linear eqations that 
we may take a basis $F_{r, s}^{(1)}, \ldots \ldots, F_{r, s}^{(k)}$ of $\mathfrak{N}_{r, s}$ over $K$ such that all the components of the tensors $F_{r, s}^{(i)}$ belong to $P$. We take such bases of $\mathfrak{N}_{r, s}$ for $r, s \leqq m$. Then a matrix $X \epsilon \mathfrak{g l}(n, K)$ is a replica of $A$ if and oniy if

$$
X_{r, s} F_{r, s}^{(i)}=0 \quad,(r, s \leqq m ; i=1,2, \ldots \ldots)
$$

But we may regard these equations as the linear equations with coefficients in $P$ of indeterminates $X=\left(x_{i j}\right)$. Then there exists $A_{i} \epsilon \mathfrak{g l}(n, P)(i=1, \ldots \ldots$, $k$ ) such that every solution $X \in \mathfrak{g l}(n, K)$ is a linear combination of $A_{i}$ with coefficients in $K$.

Lemma 6.. Let $\mathfrak{Q}$ be a Lie subalgebra of $\mathfrak{g l}(n, P)$ and $K$ an extension field of $P$. If $\mathfrak{Q}_{K}{ }^{15)}$ is l-algebraic over $K$, then $\mathfrak{L}$ is also $l$-algebraic. 'Proof. Let $X_{1}, \ldots \ldots, X_{r}$ be a basis of $\mathfrak{Q}$ over $l^{\prime}$ and $X \mathfrak{L}$. Let $X^{\prime}$ be a replica of $X$ in $\mathrm{gl}(n, P)$. Since $\mathfrak{Q}_{K}$ is l-algebraic, $X^{\prime}$ is a linear combination of $X_{1}, \ldots \ldots, X_{r}$ with coefficients in $K$. Then the same is the case with coefficients in $P$ in virtue of the same theorem of linear equations as above.

Definition $I$. Let $\mathbb{Q}$ be a Lie subalgebra of $\mathrm{gl}(n, P)$. A basis $\left\{X_{1}\right.$, $\left.\ldots \ldots, X_{r}\right\}$ of $\mathfrak{L}$ over $P$ is called algebraic if every replica of each $X_{i}$ in $\mathrm{gl}$ $\left(n, I^{P}\right)$ belongs to $\mathbb{Q}$.

Now we may prove the following theorem.

Theorem 1.' Let P te a ficla of characteristic zero. A Lic subalgebra $\mathfrak{L}$ of $\mathrm{gl}(n, P)$ is l-algebraic if and only if $\mathbb{Q}$ has an algebraic basis.

Proof. Assume that $\mathbb{L}$ possesses an algebraic basis $X_{1}, \ldots \ldots, X_{r}$. Then by Lemma $5, X_{1}^{*}, \ldots \ldots, X_{r}$ is also an algebraic basis of $\mathfrak{L}_{K}$ over $K$, where $K$ is an algebraically closed extension of $K$. Hence we see from Lemma 6 that we may assume that $P$ is algebraically closed. First we prove the theorem in the case when $\mathfrak{L}$ has the structure $\mathfrak{L}=\mathfrak{S}+\mathbb{Z}$, where $\mathfrak{S}$ is a semi-simple ideal and 3 is the center.

Let

and

$$
X_{i}=Y_{i}+Z_{i}, \quad Y_{i} \in \mathfrak{S}, \quad Z_{i} \in \mathbb{Z}
$$

$$
Y_{i}=Y_{i}^{0}+\xi_{1} Y_{i}^{1}+\ldots \ldots+\xi_{i} Y_{i}^{k}, \quad Z_{i}=Z_{1}^{0}+\xi_{1} Z_{i}^{1}+\ldots \ldots+\xi_{k} Z_{i}^{k}
$$

be the canonical decompositions of $Y_{i}$ and $Z_{i}$ respectively ${ }^{16)}$. From $\left[Y_{i} Z_{i}\right]$ $=Y_{i} Z_{i}-Z_{i} Y_{i}=0$ we get by Lemma $1\left[Y_{i}^{s} Z_{i}^{t}\right]=0$ for $s, t=0,1, \ldots \ldots, k$. Then we see that the decomposition

$$
X_{i}=I_{i}+Z_{i}=\left(Y_{i}^{0}+Z_{i}^{0}\right)+\xi_{1}\left(Y_{i}^{1}+Z_{i}^{1}\right)+\cdots \cdots+\xi_{k}\left(Y_{i}^{k}+Z_{i}^{k}\right) .
$$


is a canonical decomposition of $X_{i}$. Let $Z_{i}^{\prime}$ be a replica of $Z_{i}$ and

$$
Z_{i}^{\prime}=\alpha_{0} Z_{i}^{0}+\mu_{1} Z_{i}^{1}+\ldots \ldots+\alpha_{k} Z_{i}^{k}
$$

Then $Y_{i}^{\prime}=\alpha_{0} Y_{i}^{0}+\alpha_{1} Y_{i}^{1}+\ldots . .+\alpha_{k} Y_{i}^{k}$ and $X_{i}^{\prime}=\alpha_{0}\left(Y_{i}^{0}+Z_{i}^{0}\right)+\alpha_{1}\left(Y_{i}^{1}+Z_{i}^{1}\right)+\ldots$ $\ldots+\alpha_{k}\left(Y_{i}^{k}+Z_{i}^{k}\right)$ are replicas of $Y_{i}$ and $X_{i}$ respectively. Since' $\subseteq$ is semisimple, $\mathfrak{S}$ is $l$-algebraic ${ }^{17)}$, whence $Y_{i}^{\prime}$ $\mathfrak{S}$. But by assumption $X_{i}^{\prime}$ belongs also to $\mathfrak{Z}$ and so $Z_{i}^{\prime}=X_{i}^{\prime}-Y_{i}^{\prime} \in \mathfrak{l}$. Thus every replica of $Z_{i}$ belongs to $\mathfrak{L}$. Now let $X=Y+Z \in \mathbb{R}$. Then from $[Y, Z]=0$ we see that every replica $X^{\prime}$ of $X$ is of the form $Y^{\prime}+Z^{\prime}$, where $Y^{\prime}$ and $Z^{\prime}$ are replicas of $Y$ and $Z$ respectively. Since $X$ is of the form $X=\sum \alpha_{i} X_{i}$, it follows that $Z=\sum \alpha_{i} Z_{i}$. We see again from the commutativity of $Z_{i}^{\prime}$ 's that $Z^{\prime}=\sum \alpha_{i} Z_{i}^{\prime}$, where $Z_{i}^{\prime}$ is, for each $i$, a replica of $Z_{i}$. Therefore $Z^{\prime} \mathfrak{\&}$. But since $\mathfrak{S}$ is $l$-algebraic, $Y^{\prime} \in \mathfrak{S}$ and hence $X^{\prime}=Y^{\prime}+Z^{\prime} \mathfrak{L}$. Thus $\mathfrak{L}$ is $l$-algebraic. Next let $\mathbb{L}$ be an arbitrary Lie algebra possessing an algcbraic basis. Let $\Re_{1}$ be the radical of the derived algebra $\mathfrak{L}^{\prime}$ of $\mathfrak{R}$. Then $\mathfrak{R}_{1}$ is composed only of nilpotent matrices. Let $\mathfrak{I}_{r, s}$ be the space of $r$-times contravariant and $s$ times covariant tensors and $\mathfrak{R}_{r, s}$ the totality of tensor invariants of $\mathfrak{R}_{1}$ in $\mathfrak{I}_{r, \boldsymbol{s}}$. Since $\mathfrak{R}_{1}$ is an ideal of $\mathfrak{Q}, \mathfrak{R}_{r, 8}$ is invariant under $\mathfrak{L}$.

Let

$$
\mathfrak{P}=\sum \mathfrak{T}_{r, s}, \quad \mathfrak{Q}=\sum \mathfrak{N}_{r, s}
$$

where direct summations are extended to sufficiently high orders of $\dot{r}, s$. $\Omega$ is an invariant space under $\mathfrak{Z}$ and we denote by $(\mathbb{Q})$ the representation of $\mathfrak{Z}$ induced in $\mathfrak{Z}$. Then, by Lemma $3,(A)=0$ if and only if $A \in \mathfrak{R}_{1}$. If

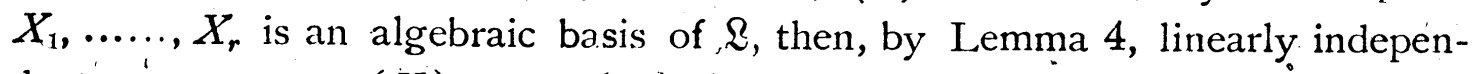
dent ones among $\left(X_{1}\right), \ldots \ldots,\left(X_{r}\right)$ form an algebraic basis of ( $(\mathbb{)})$. So ( $(\mathbb{)})$ has also an algebraic basis. On the other hand

$$
(\mathfrak{L}) \cong \mathfrak{L} / \mathfrak{R}_{1}, \quad \mathfrak{R}_{1}=\mathfrak{L}^{\prime} \cap \mathfrak{R} .
$$

$\mathfrak{R} / \mathfrak{R}_{1}$ is the center of $\mathbb{L} / \mathfrak{R}_{1}$, since $[X, Y] \in \mathfrak{R}_{1}$ for $X \in \mathfrak{Q} ; \quad Y \in \mathfrak{R}$. Hence $(\mathbb{R})$ is the direct sum of a semi-simple ideal and its center, whence $(\mathbb{R})$ is l-algebraic by the above consideration. Now let $X \in \mathbb{Q}$ and $X^{\prime}$ a replica of $X$. Then, by Lemm 4, $\mathfrak{Q}$ is invariant under $X^{\prime}$ and $\left(X^{\prime}\right)$ is a replica of $(X)$. Since $(\mathbb{R})$ is l-algebraic, there exists $Y \in \mathbb{Q}$ such that $\left(X^{\prime}\right)=(Y)$. Then $\left(X^{\prime}-Y\right)=0$, whence $X^{\prime}-Y\left(\Re_{1}\right.$ by Lemma 3 . Hence $X^{\prime} \mathfrak{Q}$ and $\mathbb{Q}$ is $l$-algebraic. The converse proposition is obvious.

From Lemma 5,6 and Theorem 1 we get the following. 
Corollary. Let $P$ be a field of characteristic zero, $\mathbb{Q} \cdot$ a Iie subalgebra of $\mathfrak{g l}^{\prime}(n, P)$ ' and $K$ an extension field of $P$. Then $\mathfrak{R}$ is $l$-algebraic if and only if $\mathbb{R}_{K}$ is an $l$-algebraic subalgebra of $\mathfrak{g l}(n, K)$.

Definition 2. A basis $\left\{X_{1}, \ldots \ldots, X_{r}\right\}$ of a Lie subalgebra $\mathfrak{Q}$ of $\mathrm{gl}(n, P)$ is called rational if each $X_{i}$ is an $n$-matrix or an $r$-matrix.

Since every replica of $n$ - or $r$-matrix is a scalar multiple of it, a rational bas!s of $\mathfrak{L}$ is an algebraic basis.

Theorem 2. Let $\mathfrak{Q}$ be a Lic subalgebra of $\mathrm{gl}(n, P)$ and $I$ an algebraically closed field of characteristic zero. Then $\mathfrak{R} \cdot$ is l-algcbraic if and only if \& has rational basis.

Proof. If $\mathfrak{Q}$ has a rational basis, then $\mathfrak{Q}$ is $l$-algebraic by Theorem 1 . Let conversely $\&$ be $l$-algebraic Lie algebra of $r$ dimensions and $X_{1}, \ldots \ldots$, $X_{m}(m>r)$ be linearly independent matrices of $\mathbb{Q}$ which are $n$ - or $r$-matrices. Let $A$ be a matrix of $\mathfrak{Z}$ which is linearly independent of $X_{1}, \ldots, \ldots, X_{m}$ and

$$
A=A^{0}+\xi_{1} A^{\prime}+\ldots \ldots+\xi_{k} A^{k}
$$

a canonical decomposition of $A$. Since $\mathfrak{L}$ is $l$-algebraic, $A_{i}(i=0,1, \ldots \ldots$, $k$ ) belong to \&. As $A$ is linearly independent of $X_{1}, \ldots \ldots, X_{m}$, at least one of them, say $A^{i}$, must be linearly independent of $X_{1}, \ldots \ldots, X_{m}$. Putting $A^{i}=X_{m+1}$, we obtain $n+1$ linearly indedendent matrices, which are $n$ - or $r$-matrices. Repeating this process we conclude that $\mathfrak{Q}$ has a rational basis.

2. Theorem 3. A Lic subalgebra $\mathfrak{Q}$ of $\mathfrak{g l}(n, P)$ is l-algebraic if and only if ils radical $\mathfrak{R}$. is l-algebraic.

Proof. Let $\mathfrak{R}$ be l-álgebraic. By Levi's theorem there exists a semisimple subalgebra $\mathfrak{S}$ of $\mathbb{R}$ such that $\mathbb{L}=\mathfrak{S}+\mathfrak{R}, \mathfrak{S} ; \mathfrak{R}=0$. Then from the $l$-algebraicity of $\mathfrak{S}$ and $\mathfrak{R}$ we see that $\mathfrak{L}$ has an algebraic basis. Therefore $\mathfrak{L}$ is älso $l$-algebraic by Theorem 1 . Let, conversely, $\mathfrak{Q}$ be $l$-algebraic. We may assume that $P$ is algebraically closed. Let $\mathfrak{R}_{1}, \mathfrak{P}, \mathfrak{Z}$ and $(\mathfrak{L})$ have the same meanings as in the proof of Theorem 1 . Then

$$
(\mathfrak{R}) \cong \mathfrak{L}^{\prime} / \mathfrak{R}_{1}+\mathfrak{R} / \mathfrak{R}_{1}
$$

where $\mathfrak{L}^{\prime} / \mathfrak{R}_{1}$ is semi-simple and $\mathfrak{R} / \mathfrak{R}_{1}$ is the center. Hence

$$
(\mathfrak{Q})=\left(\mathfrak{\Omega}^{\prime}\right)+(\mathfrak{R}) \text {, }
$$

where $\left(\mathfrak{R}^{\prime}\right)$ and $(\mathfrak{R})$ are the representations of $\mathfrak{L}^{\prime}$ and $\mathfrak{R}$ respectively and $(\mathfrak{R})$ is the center of $(\mathfrak{Q})$. By Lemma $4(\mathfrak{R})$ is l-algebraic, whence $(\mathfrak{R})$ 
is $l$-algebraic by Lemma 1 . Let $R \in \mathfrak{R}$ and $S$ be a replica, of $R$. Then $(S)$ is a replica of $(R)$. Hence $(S) \in(\Re)$ and so $S$ belongs to $\Re$. Thus $\Re$ is $l$-algebraic,

Lemma 7. Let $\mathfrak{L}$ be a Lie subalgebra of $\mathfrak{g l}(n, P)$ and $D_{A}$ the inner derivation of $\mathbb{Q}$ defined by an element $A \in \mathbb{Q}$, i.e. $\left.D_{A} X={ }_{L} A X\right]$ for $X \in \mathbb{Q} . \quad D_{A}$ is represented by $n$-matrix or $s$-matrix according as $A$ is $n$-matrix or $s$ matrix.

We omit the proof.

Lemma 8. Let $A \in \mathrm{gl}(n, P)$. Then there exists an $n$-matrix $A^{0}$ and an $s$-matrix $A^{s}$ in $g l(n, P)$ sueh that $\left[A^{0} A^{s}\right]=0$ and $A=A^{0}+A^{s}$. Moreover they are replicas of $A^{18)}$.

Proof. Let $K$ be the splitting field of the characteristic equation of $A$. Then, as we may easily verify, $A$ is decomposed uniqualy in $\mathfrak{g l}(n, K)$ into the form $A=A^{0}+A^{s}$, where $A_{0}$ and $A^{s}$ are $n$ - and $s$-matrix respectively such that $\left[A^{0} A^{s}\right]=0$. Now let $\sigma$ be any substitution of the Galois group of $K / P$. Then

$$
A=\sigma A=\sigma A^{0}+\sigma A^{s}
$$

where $\sigma A^{0}$ and $\sigma A^{s}$ are also $n$ - and $s$-matrix respectively and $\left[\sigma A^{0}, \sigma A^{s}\right]=0$. Hence, by the uniqueness of such decomposition, we get

$$
\sigma A^{0}=A^{0}, \quad \sigma A^{s}=A^{s} .
$$

This shows thar $A^{0}$ and $A^{s}$ belong to $\mathfrak{g l}(n, P)$.

Theorem $4^{19)}$. Lei $\mathbb{Q}$ be an l-algebraic Lie subatgebra of $\mathfrak{g l}(n, P)$ and $\Re$ its radical. Let $\mathbb{R}=\mathfrak{S}+\mathfrak{R}(\mathfrak{S} \cap \mathfrak{R}=0)$ be a Levil decomposition of $\mathfrak{L} ; \mathfrak{S}$ being a semi-simple subalgebra of $L$. Denote by $\mathfrak{R}$ the, ideal composed of all nilpotent matrices of $\Re$. Then there exists an l-algebraic abelian subalgebra $\mathfrak{U}$ of $\mathfrak{R}$ composed only of s-matrices such that

$$
[\mathfrak{S}, \mathfrak{A}]=0, \quad \mathfrak{R}=\mathfrak{A}+\mathfrak{N} \quad \mathfrak{A} \cap \mathfrak{l}=0 .
$$

Proof. If we regard $\mathfrak{R}$ as an $\mathfrak{S}$-module, then $\mathfrak{M}$ is an $\mathfrak{\subseteq}$-submodule. By the well known completely reducibility of the representations of semisimple Lie algebras we have $\mathfrak{R}=\mathfrak{M}+\mathfrak{R}$, where $\mathfrak{M}$ is an $\mathfrak{S}$-submodule. But by Lemma $2[\mathfrak{S}, \mathfrak{R}] \subseteq \mathfrak{L}^{\prime}: \mathfrak{R} \subseteq \mathfrak{R}$, whence $[\mathfrak{S}, \mathfrak{M}]=0$. Let $A$ be a regular element of $\Re$, i.c. an element which has as many different characteristic roots as a general element of $\mathfrak{R}$, and let $A=M+N, M \in \mathfrak{M}, N \in \mathfrak{R}$. Then $M$ is also regular. Further let $M=M^{0}+M^{s}$ be the decompositioe of $M$ as 
in Lenma 8. Since $\mathfrak{R}$ is $l$-algebraic, $M^{\mathfrak{s} \in \mathfrak{R}}$ and it is also regular. Moreover $[\mathfrak{S}, M]=0$ implies $\left[\mathfrak{S}, M^{s}\right]=0$ by Lemma 1 . Thus there exists a regular element $A$ of $\Re$ such that $A$ is $s$-matrix and $[\subseteq, A]=0$. Now we decompose $\mathfrak{R}$ by $D_{A}$ :

$$
\mathfrak{R}=\mathfrak{R}_{0}+\mathfrak{R}_{1},
$$

where $\mathfrak{R}_{0}$ is composed of all $X € \Re$ such that $D_{A}^{m} X=0$ for a sufficiently large integer $m$ and $D_{A} \Re_{1}=\Re_{1}$. As is well known, $\Re_{0}$ is a nilpotent subalgebra. Since $A$ is $s$-matrix, $D_{A}$ has simple elementary divisors by Lemma 7, whence $D_{A} X=[A X]=0$ for $X\left(\mathfrak{R}\right.$. Then by Lemma $1 \mathfrak{R}_{0}$ is $l$-algebraic. Denote by $\mathfrak{A}$, the set of all $s$-matrices, in $\mathfrak{R}_{0}$ and by $\mathfrak{R}_{0}$ the ideal of all $n$ matrices in $\Re_{0}$. Since $\mathfrak{R}_{0}$ is nilpotent, we have by Lemma $7[B X]=0$ for $B \bullet \mathfrak{A}, X \mathfrak{X} \mathfrak{R}_{0}$. Hence $\mathfrak{X}$ is an abelian ideal of $\mathfrak{R}_{0}$ and, as we may easily see, l-algebraic. Let $X\left(\Re_{0}\right.$ and $X=X^{0}+X^{s}$ as in Lemma 8 . Since $\mathfrak{R}_{0}$ is l-algebraic, $X^{0}$ and $X^{s}$ belong to $\mathfrak{R}_{0}$ and in fact $X^{\boldsymbol{s}_{\mathfrak{c}}} \mathfrak{A}, X^{0} \mathfrak{N}_{0}$. Hence $\mathfrak{R}_{0}=\mathfrak{A}+\mathfrak{N}_{0}$. . As we may readily verify $\lfloor\mathfrak{S}, A]=0$ implies $\left[\mathfrak{S}, \mathfrak{R}_{0}\right] \subseteq \mathfrak{R}_{0}$. Since $\mathfrak{N}_{0}=\mathfrak{R}_{0} \quad \mathfrak{N}$ is an $\mathfrak{S}$-submodule of $\mathfrak{R}_{0}, \mathfrak{R}_{0}=\mathfrak{I}+\mathfrak{N}_{0}$, where $\mathfrak{I}$ is an $\mathfrak{S}$ module such that $[\mathfrak{S}, \mathfrak{T}]=0$. Let $A_{1}, \ldots, \ldots, A_{m}$ be a basis of $\mathfrak{A}$. Then we may find $N_{i} \in \mathfrak{N}_{0}$ such that $A_{1}+N_{1}, \ldots \ldots, A_{m}+N_{m}$ is a basis of $\mathfrak{T}$. But since $\left[A_{i}, N_{i}\right]=0$ and $A_{i}, N_{i}$ are respectively $s$ - and $n$-matrices, $A_{i}$ and $N_{i}$ are replicas of $A_{i}+N_{i}$. Hence, by Lemma $1,\left[\mathfrak{S}, A_{i}+N_{i}\right]=0$ implies $\left[\mathfrak{S}, A_{i}\right]$ $=0$. Thus $[\mathfrak{S}, \mathfrak{A}]=0$. Moreover $\mathfrak{R}_{1}=\left[A, \mathfrak{R}_{1}\right] \subseteq[\mathfrak{R}, \mathfrak{R}] \subseteq \mathfrak{N}$. Therefore $\mathfrak{N}_{0}+\mathfrak{R}_{1}=\mathfrak{M}$ and $\mathfrak{R}=\mathfrak{U}+\mathfrak{M}$.

Remark. Any subalgebra $\mathfrak{L}$ of $\mathfrak{g l}(n, P)$ which has the structure as in the Theorem 4 has an algebraic basis and therefore $l$-algebraic.

3. Definition 3. A I ie algebra is called algebraic, if its adjoint representation is l-aglgebraic. A complex Lie group is called algebraic, if parameters of the group can be so chosen that the functions which define the multiplication of group elements are algebraic functions.

Lemma 9. The integrated group (\$) of any l-algebraic Lie subalgebra

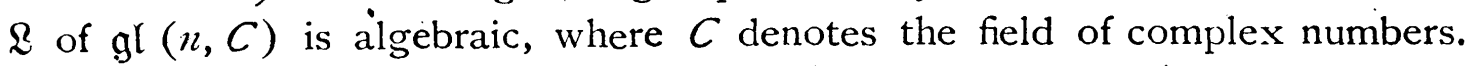

Proof. By Theorem $3, \mathbb{Q}$ has a rational basis $X_{1}, \ldots \ldots, X_{r}$. Then the matrix $G \in(S)$ which is sufficiently near the unit matrix may be represented uniquely in the form

$$
G=\exp \approx_{1} X_{1} \ldots \ldots \exp \approx_{r} X_{r},
$$

where $z_{1}, \ldots \ldots, z_{r}$ are the complex parameters. If $X_{i}$ is $n$-matrix, then we 
see easily that the coefficients of $\exp z_{i} X_{i}$ are polynomials of $z_{i}$. In case $X_{i}$ is $r$-matrix, we may assume without loss of generality that its characteristic roots are all rational integers. Then the coefficients of exp, $\approx_{i} X_{i}$ are rational functions of $\exp z_{i}$. If we change the paramster $z_{i}$ into $z_{i}$ by the equation

$$
\exp z_{i}=1+\tau v_{i}
$$

then the coefficients of $\exp z_{i} X_{i}$ are rational functions of $\tau_{i}$. Hence the coefficients of $G$ are rational functions of the suitably chosen parameters. From this we may easily verify that (S) is an algebraic group.

Since any semi-simple Lie algebra is, isomorphic with an $l$-algebraic Lie algebra, we see that any complex semi-simple lie group is algebraic.

Lemma $I O^{20)}$. Let $\mathfrak{Q}$ be algebraic and $\Re$ its radical. By Levi's theorem $\mathfrak{L}=\mathfrak{S}+\mathfrak{R}, \mathfrak{S} \cap \mathfrak{R}=0$, where $\mathfrak{S}$ is a semi-simple subalgebra of $\mathfrak{L}$. Denote by $\mathfrak{N}$ the largest nilpotent ideal. Then there exists an abelian subalgebra $\mathfrak{A}$ of $\mathfrak{R}$ such that

$$
[\subseteq, \mathfrak{A}]=0, \quad \mathfrak{R}=\mathfrak{A}+\mathfrak{N}, \quad \mathfrak{U} \cap \mathfrak{R}=0
$$

and $\mathfrak{U}$ is represented faithfully in the adjoint representation of $\mathfrak{L}$ by $l$ algebraic Lie algebra composed of 's-matrices.

Theorem 5. The Lie group (S) of any algebraic Lic algebra \& over the complex number field $C$ is algebraic.

Proof. Let $\mathfrak{L}=\mathfrak{S}+\mathfrak{U}+\mathfrak{N}$ be the direct decomposition of $\mathfrak{R}$ as in the above lemma and put $\mathfrak{I}=\mathfrak{S}+\mathfrak{A}$. Then $\mathfrak{I}$ is a subalgebra of $\mathfrak{R}$ and $\mathfrak{A}$ is

' its center. We denote $\boldsymbol{s}_{y} \underset{\mathfrak{I}_{0}}{ }$ the set of all elements $X\left(\mathfrak{T}\right.$ such that $\left[X^{\mathfrak{l}}\right]$ $=0$. Then as we may easily see, $\mathfrak{I}_{0}$ is an ideal of $\mathfrak{L}$ and is a direct factor of $\mathfrak{I}$, since $\mathfrak{I}$ is completely reducible. Let $\mathfrak{I}=\mathfrak{I}_{0}+\mathfrak{I}_{1}$. Then $\mathbb{L}=\dot{\mathfrak{I}}_{0}$ $+\mathfrak{I}_{1}+\mathfrak{N}$ and $\mathfrak{I}_{1}+\mathfrak{N}$ is obviously an ideal of $\mathfrak{L}$. $\mathfrak{I}_{0}$ is, just as $\mathfrak{I}$, direct sum of a semi-simple ideal and its center. But since $\mathfrak{I}_{0}$ is a direct factor of $\mathfrak{R}$, its center must be contained in the center 3 of $\mathfrak{R}$. As $\mathbb{Z} \subset \mathfrak{N}, \mathfrak{N} \cap \mathfrak{I}_{0}$ $=0, \mathfrak{T}_{0}$ must be semi-simple. We see readily that $\mathfrak{L}_{1}=\mathfrak{I}_{1}+\mathfrak{N}$ is also algebraic and $\mathfrak{L}=\mathfrak{I}_{0}+\mathfrak{\Omega}_{1}$. Since the Lie group of $\mathfrak{L}$ is the direct product of the Lie groups of $\mathfrak{I}_{0}$ and $\mathfrak{L}_{1}$ and any complex semi-simple group is algebraic, it is sufficient to prove the theorem in the case $\mathfrak{I}_{0}=0$. $\mathfrak{N}$ may be regarded as a representation module of $\mathfrak{I}$ and this representation $\mathfrak{D}$ of $\mathfrak{I}$ is, by our assumption, faithful and $l$-algebraic, We denote by $\mathfrak{S}$ and $\mathfrak{\Omega}$ the (local) subgroups of $\mathfrak{S}$ whose Lie algebras are $\mathfrak{N}$ and $\mathfrak{I}$ respectively. 
Then $\mathfrak{S}$ is a nilpotent invariant subgroup of $\mathbb{B}$. Every element of $\mathbb{S}$ which is sufficiently near the unit element may be represented uniquely in the form $\sigma_{a} \tau_{b}$, where $\sigma_{a} t \mathfrak{S}, \tau_{b} \in \Re$ and $(a),(b)$ denote the parameters of the groups $\mathfrak{S}$ and $\Re$ respectively. Then

$$
\left(\sigma_{a} \tau_{b}\right)^{\prime}\left(\sigma_{x} \tau_{y}\right)=\sigma_{x^{\prime}} \tau_{y^{\prime}}
$$

where

$$
\sigma_{x^{\prime}}=\sigma_{a}\left(\tau_{b} \sigma_{x} \tau_{b}^{-1}\right), \quad \tau_{y^{\prime}}=\tau_{b} \tau_{y} .
$$

We may take canonical parameters $(x)$ of the nilpotent group $\mathfrak{S}$ such that $x_{i}^{\prime}$ are polynomials of $(a)$ and $(x)$, where $\sigma_{x^{\prime}}=\sigma_{a} \sigma_{x}^{21)}$. Let $\sigma_{f(x)}=\sigma_{b}^{\prime} \tau_{x} \sigma_{b}^{-1}$. Then, as the parameters $(x)$ are canonical,

$$
f_{i}(x)=\sum_{k} \alpha_{i k}(b) x_{k} .
$$

Since the matrix $\left(a_{i k}(b)\right)$ may be represented in the form $\exp D, D \in \mathscr{D}$ and $\mathfrak{D}$ is $l$-algebraic faithful representation of $\mathfrak{T}, \Delta_{i k}(b)$ are ${ }^{\circ}$ rational functions of $(b)$ if the parameters $(b)$ are chosen suitably. Hence $x_{i}^{\prime}$, which are determined by (3), dèpend algebraically on $(a),(x)$ and (b). On the other hand, since we have chosen the parameters $(b)$ of $\Re$ as above, $y_{i}^{\prime}$ which are also defined by (3), depend algebraically on $(b)$ and $(y)$. Therefore (\$) is an algebraic group.

Let, conversely, $(\mathbb{S}$ be an algebraic Lie group and $\mathbb{R}$ its Lie algebra. It has been proved by L. Maurer ${ }^{22)}$ that the adjoint representation of $\mathfrak{L}$ has a rational basis. Hence $\mathfrak{L}$ is algebraic. Thus we obtain the following results: A complex Lie group $(S)$ is algebraic if and on'y. only if its Lie algebra $\mathfrak{R}$ is algebraic.

\section{Bibliography.}

E. Carian :

[1]. Thèse, Paris (1894).

[2] Les groupes de transformations, continus, infinis, simples, Ann. Ec. Norm. Sup. t. 26 (1909).

[3] Les representations linéaires des groupes de Lie., Jour. de Math. pures et appliquées, t. 17 (193S).

C. Chevalley:

[1] A new kind of relationship between matrices, Amer. J. math. 65 (1943).

C. Chevalley and H. F. Tuan :

[1] On algebraic Lie algebras, Proc. Nat. Acad. Sci. U.S.A. 31 (194ñ). 
M. Gơô :

[1] On the replicas of nilpotent matrices, forthcoming in Proc. Imp. Acad. Tokyo.

[2] On algebraic Lie algebras, forthcoming in this journal.

S. Lie and F. Enger :

[1] Theorie der Transformationsgruppen Bd. III (1893).

Y. Matsushima :

[1] Note on the replicas of matrices, forthcoming in Proc. Imp. Acad. Tokyo. L. Maurer :

[1] Über kontinuierliche Transformationsgruppen, Math. Ann. 39 (1891).

Mathemathical Institute
Revised December 11, 1947. Nagoya University.

\section{References.}

1) C. Chevalley and H., F. Tuan [1].

2) For the notion of replicas, see C. Chevalley [1], Cf. M. Gotô [1], Y. Matsushima [1].

3) Our l-algebraicity is the same as the algebraicity in Chevally and Tuan's sense.

4) M. Gotô [2]. M. Gotô proved also our Theorem 3 and 4 in a different waý.

5) L. Maurer [1], S. Lie and F. Engel [1] p. 800-807.

6) Y. Matsushima [1].

7) I owe this remark to $M^{-}$Gotô. below.

8) At and $B^{s}$ are commutative $(t=1, \ldots, k ; s=1, \ldots \ldots, j)$. Cf. the proof of I.emma 1

9) See. Y. Matsushima [1].

10) As usual $\mathfrak{l}_{K}$ is a $\mathrm{L}$ ie algebra over $K$ whos 2 elements are all linear combinations $a=\lambda_{1} e_{1}+\ldots \ldots+\lambda_{r} e_{r}$, where $\lambda_{i} \in K$ and $e_{1}, \ldots \ldots, e_{r}$ is a basis of $\&$ over $P$.

11) E. Cartan [2].

12) E: Cartan [1] p. 108.

13) M. Gơtô [1].

14) Y. Matsushima [1].

15) If $X_{1}, \ldots \ldots, X_{r}$ is a basis of $\mathfrak{2}$ over $P$, then $Y_{1}, \ldots \ldots, X_{r}$ are also linearly independent over $K^{-}$.

16) Some of $Y_{i} j$ and $Z_{t}^{j}$ may be zero matrices.

17) C. Chevalley and II. F. Tuan [1], M. Gotô [2].

18) Cf. Y. Matsushima [1].

19) C. Chevalıey and H. F. Tuan [1], M. Gotô [2].

20) M. Gotô [2].

21) E. Cartan [3].

22) L. Maurer [1], S. Lie and F. Engel [1] p. $800-807$ 